\title{
Validation of the clinical, radiological, biochemical parameters of osteomalacia
}

\begin{abstract}
Background: Osteomalacia, a metabolic bone disorder characterized by softening of the bones caused by defective bone mineralization secondary to inadequate amounts of available phosphorus and calcium, or because of overactive resorption of calcium from the bone as a result of hyperparathyroidism. The diagnosis of Osteomalacia is dependent on clinical, radiographic and biochemical parameters still, none of these are pathognomonic of the disorder, and histologic examination of a bone biopsy, an invasive procedure is usually necessary to confirm the diagnosis. We used diagnostic criteria based on clinical, biochemical and radiological parameters to diagnose Osteomalacia with adequate accuracy.
\end{abstract}

Methodology: This was Cross sectional and observational study. A total of 100 patients age between 18-65 years attending OPD with complaints of persistent, nonspecific and generalized musculoskeletal pain were included in the study. Thorough history was taken and examination was done. They were subjected to biochemical investigation including haemogram, serum vitamin $\mathrm{D}$, serum calcium, serum phosphorus, serum alkaline phosphates, serum PTH and radiological investigation including X-ray: Pelvis with both hips, x-ray lumbosacral spine. We used scoring criteria including pain score and Diet criteria and diagnostic index of 1 for diagnosing Osteomalacia. Data received from biochemical test was analyzed and correlated with the clinical findings.

Results: We observed that only generalized pain at presentation and vitamin D level of $<19.23 \mathrm{ng} / \mathrm{ml}$ was significantly associated with outcome. On evaluating the combination of presence of these criteria we observed it to be $75 \%$ sensitive and $93.8 \%$ specific with a PPV of $75 \%$ and NPV of $93.8 \%$ and an accuracy of $68.8 \%$.

Conclusion: Modified two component criteria can be used as for assessment, however, its efficacy against biopsy proven cases of Osteomalacia needs further evaluation apart from its validation in different population groups.

Keywords: Osteomalacia, Modified diagnostic criteria
Volume I Issue 2 - 2014

\author{
Dharmendra Kumar,' Sudhir Singh,' Rajat \\ Singh,' Nilam Bhasker,' Nishant Raj Sharma,' \\ Alok Kumar Yadav' \\ 'Department of Orthopaedics, Era's Lucknow Medical College, \\ INDIA \\ ${ }^{2}$ Department of Pathology, Era's Lucknow Medical College, \\ INDIA
}

Correspondence: Dharmendra Kumar, Department of Orthopaedics, Era's Lucknow Medical College, Lucknow, India, Emaildharmendra007I5@gmail.com

Received: October 22, 2014| Published: October 30, 2014

\section{Introduction}

Osteomalacia is the softening of the bones caused by defective bone mineralization secondary to inadequate amounts of available phosphorus and calcium, or because of overactive resorption of calcium from the bone as a result of hyperparathyroidism (which causes hypocalcaemia, in contrast to other etiologies). Osteomalacia, a metabolic bone disorder characterized by impaired mineralization, is a rarely encountered disease in the western world. ${ }^{2-4}$ Together with rickets and osteoporosis it contributes to $0.1 \%$ of total global disease burden and $1 \%$ of the population. ${ }^{5}$ Osteomalacia is a common metabolic bone disorder in countries such as India due to suboptimal nutrition. ${ }^{67}$

Osteomalacia is considered to be an extension of rickets in adults, and because of this, use of the term Osteomalacia is often restricted to the milder, adult form of the disease. It may show signs as diffuse body pains, muscle weakness, and fragility of the bones. The most common cause of the disease is a deficiency in vitamin $\mathrm{D}$, which is normally obtained from the diet and/or from sunlight exposure. ${ }^{8}$ The radiographic appearance of Osteomalacia may be normal or similar to findings noted with osteoporosis. However, coarseness of the trabeculae may differentiate Osteomalacia from osteoporosis. The differential diagnosis of generalized osteopenia includes
Osteomalacia, hyperparathyroidism, and multiple myeloma. ${ }^{9}$ The diagnosis of Osteomalacia is dependent on clinical, radiographic and biochemical parameters still, none of these are pathognomonic of the disorder, and histologic examination of a bone biopsy, an invasive procedure is usually necessary to confirm the diagnosis. ${ }^{10}$ McKenna et al. ${ }^{11}$ Came up with a criteria based on clinical, biochemical and radiological assessment to diagnose Osteomalacia and till date remains the one of the most accepted criteria for diagnosis of Osteomalacia sans biopsy. ${ }^{9,11}$ Though, this scoring system does not include biopsy yet it is complicated to administer owing to multiple investigations and is complex to score owing to variable criteria of assigning scores to different components of the scoring criteria. We decided to develop a simplified, noninvasive, cost-effective and patient friendly diagnostic criteria based on clinical, biochemical and radiological parameters that could detect the condition with adequate accuracy. The other objective of the present work was to evaluate the clinical profile of patients with complaints of persistent, non-specific and generalized musculoskeletal pain for the epidemiological purposes.

\section{Material and methods}

This was Cross sectional and observational study was conducted in Orthopaedics Department of Era's Lucknow Medical College and Hospital over period of 18 months. The study was cleared by 
the institutional research cell and the ethical committee. Informed written consent was taken from all the subjects included in the study. A total of 100 patients above 18 years attending OPD with complaints of persistent, non-specific and generalized musculoskeletal pain were included in the study. All the patients with age more than 65 years, with known pathological causes of Osteomalacia, with known pharmacological causes of Osteomalacia, with known cases of polyarthritis were excluded from study. Thorough history was taken and examination was done. They were subjected to biochemical investigation including haemogram, serum vitamin $\mathrm{D}$, serum calcium, serum phosphorus, serum alkaline phosphates, serum PTH and radiological investigation including X-ray: Pelvis with both hips, $\mathrm{x}$-ray lumbosacral spine. Data received from biochemical test was analyzed and correlated with the clinical findings. We used scoring criteria including pain score and Diet criteria (Table 1). We used the diagnostic index of for diagnosing Osteomalacia. For the purpose of this study we used the diagnostic index of (Table 2). ${ }^{11}$

Table I Diet criteria

\begin{tabular}{ll}
\hline Type & Score \\
\hline Vegetarian & $\mathrm{I}$ \\
Non-vegetarian & 2 \\
< once a week & 4 \\
$\geq$ once a week & \\
Milk Intake & 1 \\
Infrequent & 2 \\
Daily I glass & 3 \\
Daily 2 glass or more & \\
\hline
\end{tabular}

Total Diet Score $=$ Type Score + Milk Score

Table 2 Validation criteria

\begin{tabular}{ll}
\hline Clinical Features & Score \\
\hline Limb pain & $\mathrm{I}$ \\
Proximal Myopathy & $\mathrm{I}$ \\
Chemistry & \\
Calcium-phosphate product & \\
I.62-2.4I(m mol/I) & $\mathrm{I}$ \\
$\mathrm{I} .21-\mathrm{I} .6 \mathrm{I}$ & 2 \\
$<1.2 \mathrm{I}$ & 3 \\
Alkaline phosphatase (score only when LFT is & \\
normal) & \\
$75-100(\mathrm{IU} / \mathrm{I})$ & $\mathrm{I}$ \\
I0I-20I & 2 \\
$20 \mathrm{I}-300$ & 3 \\
$>300$ & 4 \\
$25-(\mathrm{OH})$ vitamin D & \\
$5-12.5 \mathrm{n}$ mol/I & $\mathrm{I}$ \\
$<5$ & 2 \\
Radiology & \\
Pseudo fractures & $\mathrm{I}$ \\
\hline
\end{tabular}

Interpretation of scores:
a) $>35 \%$ or Score $>5=$ Osteomalacia
b) $15-35 \%$ or Score $3-4=$ suspected Osteomalacia
c) $<15 \%$ or Score $<2=$ negative for Osteomalacia

\section{Results}

Total 100 patients were included out of which 60 were female and 40 were male patients. Mean age of patients was $31.21 \pm 8.52$ years. Lower Middle $(n=34)$, Upper Middle $(n=33)$ and Upper Lower socioeconomic classes were more commonly involved as compared to lower $(n=8)$ and upper socioeconomic class $(n=3)$. Majority of patients $(n=76)$ were in healthy weight category. There were $(n=9)$ underweight and $(n=15)$ underweight patients. Majority $(n=52)$ of patients had generalized pain followed by those having lower back pain $(n=41)$, lower limb pain $(n=34)$ and upper limb pain $(n=17)$. A total of $(n=11)$ had upper back pain and $(n=9)$ had hip pain. There were $(n=2)$ patients with pain at other locations. Almost half the patients were vegetarian $(n=46)$. There were $(n=54)$ non-vegetarians but meat consumption at least once a week or more was reported by $(n=40)$ patients only. Although $(n=98)$ patients reported of milk intake yet majority were infrequent milk consumers $(n=76)$. There were only $(\mathrm{n}=18)$ patients reporting consumption of at least one glass of milk each day and $4(n=4)$ who reported consuming more than one glass of milk every day. Almost two third (66\%) patients were anemic. Total leukocyte count $>10,000 / \mathrm{cu} \mathrm{mm}$ was recorded in 8 patients. Mean neutrophil, lymphocyte, eosinophil and monocyte count was $67.15 \pm 7.49,27.73 \pm 7.09,3.18 \pm 2.60$ and $1.95 \pm 1.64 \%$ respectively. ESR $>17 \mathrm{~mm} / \mathrm{hr}$ was recorded in 57 patients.

All the subjects had 25-(OH) vitamin D2 levels $>12.5 \mathrm{n} \mathrm{mol} / 1$. Calcium-Phosphorous product was $>2.41 \mathrm{n} \mathrm{mol} / 1$ in 13 (13\%). A total of $50(50 \%)$ patients had calcium-phosphorous product in the range of $1.62-2.41 \mathrm{n} \mathrm{mol} / \mathrm{l}$ and $32(32 \%)$ had Ca-P product in the range of 1.21-1.61 n mol/1. A total of 5 (5\%) patients had Ca-P product $<1.21$ n mol/l. S. Alkaline phosphatase levels were $<75 \mathrm{IU} / 1$ in $3(3 \%)$ patients only. Total of $22(22 \%)$ had serum ALP levels in range 75 $100 \mathrm{IU} / 1$ and 75 (75\%) had S. ALP levels in the range of 101-201 IU/1. Mean serum parathyroid hormone levels ranged from 21-80 pg/ml with a mean value of $48.35 \pm 11.84 \mathrm{pg} / \mathrm{ml}$ (Table 3). Radiographs of spine showed normal imaging in 44 cases, areas of rarefaction in 11 cases and decreased density in 45 cases. Similarly pelvis radiographs showed normal density in 47 cases, rarefaction in 8 cases, decreased bone density in 37 cases, Loser's zone in 4 cases and sclerotic iliac crest in 4 cases. Using McKenna's criteria as the basis, a total of 20 out of 100 patients with persistent non-specific musculoskeletal pain were found to be confirmed for Osteomalacia.

In present study, generalized body pain, tenderness near lumbar and/or hip area, dietary scores, hemoglobin level, 25-OH vitamin $\mathrm{D}$, serum calcium, serum phosphorous, serum alkaline phosphatase, serum PTH levels and radiological findings were significantly associated with Osteomalacia. Out of these, generalized body pain, tenderness near lumbar hip area and radiological findings were categorical in nature while dietary score, hemoglobin level, 25$\mathrm{OH}$ vitamin $\mathrm{D}$, serum calcium, serum phosphorous, serum alkaline phosphatase and serum PTH levels were continuous in nature. For the purpose of development of scoring criteria, the continuous variables were converted into categorical variables using receiver operator curve analysis. The criteria for selection of cut-off value were sensitivity above $75 \%$. The cut-off values for diet score, Hb, Vitamin D, S. Ca, S. P, S. ALP and PTH levels were $<3,<11.5,<19.23,<8.05,<2.85,>117$ and $>45.90$ respectively (Table 4 ). For clinical parameters (History and clinical examination) the criteria was $90 \%$ sensitive and $63.8 \%$ specific.

However, it had a very low PPV (positive predictive value) 
(38.3\%) and a high NPV (negative predictive value) (96.2\%). The overall efficiency (Youden's Index) was 53.8\% only. For laboratory parameters (hematological and biochemical criteria) the criteria was $95 \%$ sensitive, $55 \%$ specific, had a PPV of $34.5 \%$ and a NPV of $97.8 \%$. Overall efficiency of the criteria was $50 \%$. Similarly for radiological parameter the criteria were $80 \%$ sensitive and $63.75 \%$ specific with a very low PPV (35.56\%) and a high NPV (92.73\%). The overall efficiency (Youden's Index) was $43.75 \%$ only. Vitamin Table 3 Distribution of patients according to vitamin $\mathrm{D}$ and other biochemical levels

\begin{tabular}{|c|c|c|c|}
\hline SN & Variable & No. of Cases & Percentage \\
\hline \multirow{2}{*}{ I. } & 25-(OH) Vitamin D levels & & \\
\hline & $>12.5 \mathrm{nmol} / \mathrm{l}$ & 100 & 100.0 \\
\hline \multirow{5}{*}{2.} & Calcium-Phosphorous product & & \\
\hline & $>2.41 \mathrm{nmol} / \mathrm{l}$ & 13 & 13.0 \\
\hline & $\mathrm{I} .62-2.4 \mathrm{Imol} / \mathrm{l}$ & 50 & 50.0 \\
\hline & $1.21-1.611 \mathrm{nmol} / \mathrm{l}$ & 32 & 32.0 \\
\hline & $<1.21 \mathrm{nmol} / \mathrm{l}$ & 5 & 5.0 \\
\hline \multirow{4}{*}{3.} & Serum Alkaline phosphatase & & \\
\hline & $<75 \mathrm{IU} / \mathrm{I}$ & 3 & 3.0 \\
\hline & $75-100 \mathrm{IU} / \mathrm{I}$ & 22 & 22.0 \\
\hline & I0I-20| IU/I & 75 & 75.0 \\
\hline 4. & Mean S. PTH \pm SD levels (Range) pg/ml & $48.35 \pm 11.84(2$ & \\
\hline
\end{tabular}

Table 4 Cut-off variables for variables found to be significantly associated with Osteomalacia

\begin{tabular}{llll}
\hline Variable & Selected cut-off & \multicolumn{2}{c}{ Independent Efficacy } \\
& & Sensitivity & Specificity \\
\hline Diet score & $\leq 3$ & 80 & 65 \\
$\mathrm{Hb}(\mathrm{gm} / \mathrm{dl})$ & $\leq 11.5$ & 75 & 62.5 \\
Vitamin D $(\mathrm{ng} / \mathrm{ml})$ & $\leq 19.23$ & 90 & 70.0 \\
S. Ca $(\mathrm{mg} / \mathrm{dl})$ & $\leq 8.05$ & 80 & 50 \\
S.P $(\mathrm{mg} / \mathrm{dl})$ & $\leq 2.85$ & 75 & 62.5 \\
S.ALP $(\mathrm{IU} / \mathrm{L})$ & $\geq 117$ & 85 & 61.2 \\
PTH $(\mathrm{pg} / \mathrm{ml})$ & $\geq 45.90$ & 75 & 48.7 \\
\hline
\end{tabular}

Table 5 Comparison of various parameters in diagnosis of Osteomalacia

D parameter showed a sensitivity of $75 \%$, specificity of $93.8 \%$, PPV $75 \%$ and NPV $93.8 \%$ with an accuracy of $68.8 \%$ (Table 5).

After binary regression, we observed that only generalized pain at presentation and vitamin D level of $<19.23 \mathrm{ng} / \mathrm{ml}$ was significantly associated with outcome. On evaluating the combination of presence of these criteria we observed it to be $75 \%$ sensitive and $93.8 \%$ specific with a PPV of $75 \%$ and NPV of $93.8 \%$ and an accuracy of $68.8 \%$.

\begin{tabular}{|c|c|c|c|c|c|c|c|c|c|c|c|c|c|}
\hline & & \multicolumn{3}{|c|}{ Clinical Parameter } & \multicolumn{3}{|c|}{ Laboratory Parameter } & \multicolumn{3}{|c|}{ Radiological Parameter } & \multicolumn{3}{|c|}{ Vitamin D Parameter } \\
\hline & & Positive & Negative & Total & Positive & Negative & Total & Positive & Negative & Total & Positive & Negative & Total \\
\hline & Positive & 18 & 02 & 20 & 19 & 01 & 20 & 16 & 04 & 20 & 15 & 05 & 20 \\
\hline & Negative & 29 & 51 & 80 & 36 & 44 & 80 & 29 & 51 & 80 & 5 & 75 & \\
\hline & Total & 47 & 53 & 100 & 55 & 45 & 100 & 45 & 55 & 100 & 20 & 80 & 100 \\
\hline \multirow{6}{*}{ OSTEOMALACIA } & & & & & & & & & & & & & \\
\hline & Sensitivity & $90 \%$ & & & $95 \%$ & & & $80 \%$ & & & $75 \%$ & & \\
\hline & Specificity & $63.8 \%$ & & & $55 \%$ & & & $63.75 \%$ & & & $93.8 \%$ & & \\
\hline & PPV & $38.3 \%$ & & & $34.5 \%$ & & & $35.56 \%$ & & & $75 \%$ & & \\
\hline & NPV & $96.2 \%$ & & & $97.8 \%$ & & & $92.73 \%$ & & & $93.8 \%$ & & \\
\hline & $\mathrm{YI}$ & $53.8 \%$ & & & $50 \%$ & & & $43.75 \%$ & & & $68.8 \%$ & & \\
\hline
\end{tabular}




\section{Discussion}

On evaluating the association between age and Osteomalacia, the association was not found to be significant $(\mathrm{p}=0.467$ ). Like persistent non-specific musculoskeletal pain, Osteomalacia has also been reported to be prevalent in all age groups. The mean age of patients in the present study was $31.21 \pm 8.52$ years (range: 18 to 65 years), ${ }^{11}$ too did not find any association between age and Osteomalacia and did not include age as a component of diagnostic index developed by them. Similar results of age and Osteomalacia was found in other study. ${ }^{12}$ In their study among adolescents (10-16 years) reported the mean age of patients to be 13.2 years only. In present study, the prevalence was higher in females $(65 \%)$ as compared to males $(35 \%)$ yet the association could not be established statistically $(\mathrm{p}=0.610)$. Although prevalence of Osteomalacia has been reported to be higher in females with a female to male ratio being as high as $11.33: 1^{2}$ to $5: 1 .^{13}$ Majority of our patients were from middle (lower middle and upper middle) socioeconomic strata. Socio-economic status, a measure of deprivation, is typically classified by examination of factors such as home ownership, levels of unemployment, and education. There is an inverse relationship between socio-economic status and the prevalence of both acute and chronic pain. ${ }^{14-17}$ However, contrary to these reports, in present study, lower middle and upper middle class was more widely affected. A possible explanation for this could be association of chronic wide spread pain with physical inactivity. ${ }^{18}$

In present study, majority ( $52 \%$ ) of patients had generalized pain followed by those having lower back pain (41\%), lower limb pain (34\%) and upper limb pain (17\%). A total of 11 had upper back pain and 9 had hip pain. There were 2 patients with pain at other locations. Among patients not reporting with generalized body pain, except for 1 patient, all the patients had pain at one or more locations. More than half the patients not reporting generalized pain had involvement of three or more sites. These findings are in concordance with the observations of ${ }^{19}$ who also reported that chronic musculoskeletal pain is rarely present in a single body site. In present study, majority of patients were anemic $(66 \%)$. Thus, indicating a high prevalence of nutritional deficiency. Hypovitaminosis D has often been seen to be manifested in combination with other nutritional deficiencies like anemia. ${ }^{20}$ In present study, we evaluated serum $25(\mathrm{OH})$-vitamin D levels ${ }^{11}$ using the criteria of and found that all the patients had vitamin $\mathrm{D}$ levels more than $12.5 \mathrm{n} \mathrm{mol} / 1$. However, using the criteria proposed by Holick ${ }^{21}$ who proposed a normal range of 50-140 $\mathrm{n} \mathrm{mol} / 1$, we found exactly half the patients $(n=50)$ to be having $25-(\mathrm{OH})$ Vitamin D3 levels to be less than $50 \mathrm{nmol} / 1$, thus indicating a high prevalence of Hypovitaminosis D in the study subjects. A number of researchers have attributed Hypovitaminosis D to be the cause attributable to persistent non-specific musculoskeletal pain. ${ }^{22,23}$ However, using the criteria all the patients were seen to be having low $\mathrm{Ca}-\mathrm{P}$ product and raised Alkaline Phosphatase levels. ${ }^{11}$ Using normal reference range of PTH to be $0-40 \mathrm{IU} / \mathrm{L}$, as many as 74 cases had values above $40 \mathrm{IU} / \mathrm{L}$.

Using McKenna's criteria, a total of 20 out of 100 patients with persistent non-specific musculoskeletal pain were found to be confirmed for Osteomalacia. Osteomalacia has been reported to be a common reason for persistent non-specific muscular pain ${ }^{22}$ especially in cases with Hypovitaminosis D. In present study, we had a high prevalence of hypovitaminosis D and it could be attributed to the high prevalence of Osteomalacia in these patients. In our study, tenderness near lumbar/hip area was found to be significantly associated with
Osteomalacia $(\mathrm{p}=0.021)$. All cases were able to perform their routine tasks and were fully ambulatory, thus indicating a mild form of disease.

In present study, vegetarian or infrequent non-vegetarian diet and irregular or no milk consumption were found to be associated with Osteomalacia $(\mathrm{p}<0.05)$. Mean dietary scores were significantly lower in cases of Osteomalacia $(2.25 \pm 0.79)$ as compared to those without Osteomalacia $(3.9 \pm 1.77)(\mathrm{p}<0.001)$. A clear-cut relationship between nutritional deficiency and Osteomalacia has also been shown by others. ${ }^{24-27}$ Evaluation of hematogical parameters showed that except for mean hemoglobin levels statistically no significant difference was observed between two groups with respect to any of the hematological parameters $(\mathrm{p}<0.001)$.

Vitamin D deficiency is the most commonly reported cause of Osteomalacia and is reported in almost all the diagnostic work ups for Osteomalacia. According to Osteomalacia ${ }^{27}$ is an end-stage bone disease of chronic and severe vitamin D or phosphate depletion of any cause. For the purpose of validation, three sets of criteria were evolved - clinical and dietary criteria, hematological and biochemical criteria and radiological criteria. Three criteria were evolved as we focused on developing simplified criteria which can have accuracy level close to that of McKenna's Diagnostic Index. ${ }^{11}$

On evaluating the clinical and dietary criteria, it was found to be $90 \%$ sensitive and $63.8 \%$ specific. However, it had a very low PPV of $38.3 \%$ and a high negative predictive value of $96.2 \%$. The overall accuracy (Youden's Index) was $53.8 \%$ only. The clinical criterion is easy to be used and can decrease the burden of sophisticated investigations by around $50 \%$ and hence can be used as a pre-hospital/ self-assessment criteria with ample sensitivity (90\%). Hematological and biochemical criteria was based on estimation of hemoglobin, Vitamin D, serum Ca, S. P, S. ALP and PTH levels, thus including a spectrum of diagnostic tests which helped it to yield a highly sensitive criteria (95\%) but owing to high prevalence of anemia in Indian population resulted in a less specific outcome (55\%) and made its positive predictive value highly compromised $(34.5 \%)$. The radiological criteria independently too had the limitation of low specificity and had specificity similar to clinical and dietary criteria (63.8\%) but had a sensitivity lower than the other two criteria.

In order to improve the diagnostic efficacy of our criteria, we suggest the use of a combined criteria of two factors- generalized non-specific persistent pain on presentation and vitamin D levels $<19.23 \mathrm{ng} / \mathrm{ml}$ and found it to be highly specific $(93.8 \%)$. If all the cases diagnosed as positive by clinical and dietary criteria are also included in these criteria then it turns out of be $85 \%$ specific. This modified two-component criteria was $85 \%$ sensitive, $93.8 \%$ specific had a positive predictive value of $77.3 \%$ and a negative predictive value of $96.2 \%$ and diagnostic efficacy of $81.2 \%$. (Table 6 ). The criteria developed in present study was developed in a specific population and was validated against an already existing non-invasive diagnostic criteria, however, its efficacy against biopsy proven cases of Osteomalacia needs further evaluation apart from its validation in different population groups. If the proposed criteria emerges as a useful utility against both biopsy proven Osteomalacia cases and is applicable in various populations then it can be used as a simplified diagnostic tool with adequate accuracy, however, even in its present form it is a useful criteria for assessment of Osteomalacia and can be used in a low resource setting. 
Table 6 Comparison of various parameters in diagnosis of Osteomalacia

\begin{tabular}{|c|c|c|c|}
\hline \multirow[b]{2}{*}{ Generalized Body Pain + Vitamin D $\leq 19.23 \mathrm{ng} / \mathrm{ml}$ or Positive in Clinical Criteria } & \multicolumn{2}{|c|}{ Osteomalacia } & \multirow[b]{2}{*}{ Total } \\
\hline & Positive & $\begin{array}{l}\text { Suspect/ } \\
\text { Negative }\end{array}$ & \\
\hline Positive & 17 & 5 & 22 \\
\hline Negative & 3 & 75 & 78 \\
\hline Total & 20 & 80 & 100 \\
\hline
\end{tabular}

Sensitivity, 85\%; Specificity, 93.8\%, PPV, 77.3\%; NPV, 96.2\%;YI, 8I.2\%

\section{Conclusion}

Persistent non-specific generalized musculoskeletal pain was independent of age, more common in females, middle income groups (probably related with low physical activity), vegetarians/ occasional non-vegetarians and those who did not consume milk or consumed milk irregularly. It did not show an association with BMI, but had an association with multiple sites of pain. A high prevalence of anemia indicated the role of nutritional deficiencies in the cause-effect relationship of persistent non-specific generalized musculoskeletal pain with vitamin $\mathrm{D}$, calcium-phosphorus product, alkaline phosphatase and serum parathyroid hormone levels. The present study thus highlights the different causes of persistent nonspecific generalized musculoskeletal pain and evolved simplified two-component criteria which were close to multiple-component criteria (McKenna Diagnostic index) in terms of accuracy. Pending the evaluation of these newly evolved criteria against biopsy proven Osteomalacia cases and its utility in various population groups, the criteria can be used as a preliminary assessment tool for diagnosis of Osteomalacia in low-resource setting. Further studies for improvement, validation and reliability assessment are recommended.

\section{Acknowledgments}

None.

\section{Conflicts of interest}

The author declares that there is no conflict of interes.

\section{References}

1. McKenna MJ, Freaney R. Noninvasive testing in the diagnosis of Osteomalacia. Am J Med. 1995;99(1):107-108.

2. Chalmers J, Conacher WD, Gardner DL, et al. Osteomalacia-A common disease in elderly women. J Bone Joint Surg Br. 1967;49(3):403-423.

3. Frame B, Parfitt AM. Osteomalacia: current concepts. Ann Intern Med. 1978;89(6):966-982.

4. Francis RM, Selby PL. Osteomalacia. Baillieres Clin Endocrinol Metab. 1997;11(1):145-163.

5. Lucas RM, McMichael AJ, Armstrong BK, et al. Estimating the globa disease burden due to ultraviolet radiation exposure. Int $J$ Epidemiol. 2008;37(3):654-667.

6. Rao DS. Role of vitamin D and calcium nutrition in bone health in India In: Mithal A, et al. (Eds.), 1st edn, Metabolic Bone Disorders. Hindustani Book Depot, Lucknow, 1998; p. 71-75.

7. Gupta A. Osteoporosis in India. The nutritional hypothesis. Natl Med J India. 1996;9(6):268-274.
8. Hazzari MA, Alzeer I, Tamimi W, et al. Clinical presentation and etiology of osteomalacia/rickets in adolescents. Saudi J Kidney Dis Transpl. 2013;24(5):938-941.

9. Tigges S, Nance EP, Carpenter WA, et al. Renal osteodystrophy: imaging findings that mimic those of other diseases. AJR Am J Roentgenol. 1995;165(1):143-148.

10. Bingham CT, Fitzpatrick LA. Noninvasive testing in the diagnosis of osteomalacia. Am J Med. 1993;95(5):519-523.

11. McKenna MJ, Freaney R, Casey OM, et al. Osteomalacia and osteoporosis: evaluation of a diagnostic index. J Clin Pathol. 1983;36(3):245-252.

12. El-Desouki MI, Othman SM, Fouda MA. Bone mineral density and bone scintigraphy in adult Saudi female patients with osteomalacia. Saudi Med J. 2004;25(3):355-358.

13. Allen SC, Raut S. Biochemical recovery time scales in elderly patients with Osteomalacia. J R Soc Med. 2004;97(11):527-530.

14. Roth RS, Punch MR, Bachman JE. Educational achievement and pain disability among women with chronic pelvic pain. J Psychosom Res. 2001;51(4):563-569.

15. Portenoy RK, Ugarte C, Fuller I, et al. Population-based survey of pain in the United States: differences among white, African American, and Hispanic subjects. J Pain. 2004;5(6):317-328.

16. Brekke M, Hjortdahl P, Kvien TK. Severity of musculoskeletal pain: relations to socioeconomic inequality. Soc Sci Med. 2002;54(2):221-228.

17. Brekke M, Hjortdahl P. Musculo-skeletal pain among 40- and 45-year olds in Oslo: differences between two socioeconomically contrasting areas, and their possible explanations. Int J Equity Health. 2004;3(1):10.

18. McBeth J, Nicholl BI, Cordingley L, et al. Chronic widespread pain predicts physical inactivity: Results from the prospective EPIFUND study. Eur J Pain. 2010;14(9):972-979.

19. Carnes D, Parsons S, Breen A, et al. Chronic musculoskeletal pain rarely presents in a single body site: results from a UK population study. Rheumatology (oxford). 2007;46(7):1168-1170.

20. de Torrente de la Jara G, Pecoud A, Favrat B. Female asylum seekers with musculoskeletal pain: the importance of diagnosis and treatment of hypovitaminosis D. BMC Fam Pract. 2006;7:4.

21. Holick MF. Calcium and vitamin D. Diagnostics and therapeutics. Clin Lab Med. 2000;20(3):569-590.

22. Plotnikoff GA, Quigley JM. Prevalence of severe hypovitaminosis D in patients with persistent, nonspecific musculoskeletal pain. Mayo Clin Proc. 2003;78(12):1463-1470.

23. Knutsen KV, Brekke M, Gjelstad S, et al. Vitamin D status in patients with musculoskeletal pain, fatigue and headache: a cross-sectional descriptive study in a multi-ethnic general practice in Norway. Scand J Prim Health Care. 2010;28(3):166-171. 
24. Singer RF. Textbook of Rheumatology. In: Kelley WN, et al. (Eds.), Metabolic bone disease. Saunders, Philadelphia, USA, 1985.

25. Nield LS, Mahajan P, Joshi A, et al. Rickets: Not a disease of the past. Am Fam Physician. 2006;74 (4):619-626.
26. Wagner CL, Greer FR. Prevention of rickets and vitamin D deficiency in infants, children, and adolescents. Pediatrics. 2008;122(5):1142-1152.

27. Bhan A, Rao AD, Rao DS. Osteomalacia as a result of vitamin D deficiency. Endocrinol Metab Clin North Am. 2010;39(2): 321-331. 